\title{
Molecular Simulations of DNA Counterion Distributions
}

\author{
Alexander P. Lyubartsev \\ Stockholm University, Stockholm, Sweden
}

\section{INTRODUCTION}

One of the remarkable physical properties of a DNA molecule is that it is a strongly charged polyelectrolyte. In solution, DNA dissociates, forming a negatively charged polyion surrounded by an atmosphere of mobile, positively charged counterions. Although positive counterions are attracted to DNA, they screen the negative charge of DNA, decreasing the attractive force for other positive counterions. Additionally, ions of different valency and size interact with DNA in a different manner, leading to effects of competition between ions of different species. There is always a delicate balance of forces forming the equilibrium ion distribution around DNA. The functionality of DNA in the cell is, in a decisive degree, determined by electrostatic forces, which in turn are dependent on the presence of different charged components in the surrounding solution. It is clear that understanding of DNA functionality is impossible without an understanding of electrostatic interactions of DNA with the environment.

The aim of the present review is to show how molecular computer simulations can contribute to our understanding of the basic features of the interaction of DNA with its ionic environment, what kind of information can be obtained by computer simulations, and how this information can be used to bridge experimental and theoretical studies of DNA. First, some common polyelectrolyte models of DNA will be briefly reviewed, and a survey of available computer simulation techniques will be given. Then, applications of computer simulations to describe the ionic environment of DNA on different levels of precision will be discussed: Monte Carlo (MC) and Brownian dynamics (BD) simulations within the continuum dielectric models, molecular dynamics (MD) simulations with explicit treatment of solvent, as well as a combination of these techniques, giving rise to the "multiscale modeling' approach.

Several reviews devoted to different aspects of DNAion interactions and computer simulations of DNA have recently appeared. ${ }^{[1-3]}$ A more general and detailed review of the computer simulation of polyelectrolytes is presented in Ref. [4].

\section{DNA POLYELECTROLYTE MODELS AND THEORIES}

A theoretical description of the ionic environment of DNA is not a simple task. In fact, in any condensed matter system, the more detailed and closer to reality the molecular model is, the more complicated (and often including even more approximations) is the theory that has to be applied to obtain meaningful results. There exist several levels of theoretical descriptions of polyelectrolyte systems. In the simplest approach (the so-called "primitive model',), a DNA is presented as a rigid cylinder, and mobile ions as point charges or small rigid charged spheres. Within the primitive model, the solvent is described as a uniform dielectric continuum, described by a dielectric permittivity of this solvent. The interaction between ions is described by the Coulombic potential scaled by the value of the dielectric permittivity.

The most commonly used approach to describe the properties of polyelectrolyte solutions at this level is the Poisson-Boltzmann (PB) (mean field) theory. ${ }^{[5]}$ This approach implies a Boltzmann distribution of the mobile ion species in the average field of other ions. For a simple cylindrical geometry, the PB equation can be rather easily solved by numerical methods. If the electrostatic potential is small, the exponent term in the standard PB equation may be linearized, resulting in the linearized PB equation that allows an analytical solution. In this context, the counterion condensation (CC) model formulated by Manning $^{[6]}$ can also be mentioned. According to the CC model, for a highly charged cylindrical polyion, a certain amount of counterions remains in close proximity to the polyion. These counterions neutralize a given fraction of the total polyion charge, whereas the remaining counterions reside in the bulk phase. Because most of the charge of the polyion ( $76 \%$ in the case of DNA) is neutralized, the distribution of ions in the bulk phase may be described by the linearized PB equation. Because of its simplicity, the Manning CC model has had a profound impact on practical, as well as theoretical, studies of polyelectrolyte solutions.

The major problem with theories based on the PB approximation is that the Boltzmann expression for the ion 
density cannot be strictly derived from the statisticalmechanical theory; therefore the accuracy of the PB theory should be checked by more rigorous theories, or by simulations. The PB theory neglects the small ion correlations within the model. The effect of correlations can be explained as follows. If a counterion is present at some point near the polyion surface, it will decrease the probability for other counterions to be around it. Therefore near this point, the counterion density will be lower than that given by the PB theory. The decrease in local counterion density causes an effective attractive force (in addition to the mean force) that draws the counterions closer to the polyion surface. Clearly, the effect becomes stronger with increase of the ion valency. Ion correlation effects can considerably change the behavior of the polyelectrolyte systems in quantitative as well as qualitative ways.

There exist a number of liquid state theories, based on integral equations, which go beyond the PB approximation: the hypernetted chain approximation (HNC), ${ }^{[7,8]}$ the BBGY chain equations, ${ }^{[9,10]}$ the mean spherical approximation, ${ }^{[5]}$ the modified PB theory, ${ }^{[11]}$ as well as some others. A discussion of these theories and a comparison of predictions of such models to both the PB model and that of computer simulations can be found in a recent review. $^{[12]}$

More elaborate DNA models may include specific details of its structure. One example is the so-called "grooved" model of DNA, ${ }^{[13]}$ in which the charged groups of DNA are located outside the cylindrical hard core, on the sites corresponding to the phosphate groups of DNA. In addition, full-atomic molecular models of DNA or other polyelectrolytes in continuum solvent have been considered. In such cases, the PB equation becomes three-dimensional, but its solution is still rather straightforward. ${ }^{[14,15]}$ Sometimes effects of polarization and hydration are included by considering a distant-dependent and field-dependent dielectric constant. The relevant theory to treat this class of models is the modified PB equation. ${ }^{[16]}$ Stricter statistical-mechanical theories become too complicated to be of any practical use in this case.

The above-discussed analytical theories have to resort to approximations already at the level of the primitive model. Comparison of theory with experiment is, in such cases, inconclusive; if discrepancies occur, it may be difficult to attribute them to the approximations within the model, or to approximations during the mathematical treatment of the model. Computer simulations can help to answer the questions because they may provide an accurate, asymptotically exact, statistical-mechanical solution for a given model. Moreover, computer simulations allow to treat even more accurate and detailed models, with a proper account of effects caused by hydration, dielectric saturation, and the molecular structure of the solvent by considering explicit solvent molecules.

The main computer simulation methods are $\mathrm{MC}$ and MD. The MC method ${ }^{[17]}$ is based on a stochastic procedure, which generates molecular configurations with probabilities equal to that in the canonical (or another statistical) ensemble. By calculating simple arithmetic averages over generated molecular configurations, it is possible to define average values of physical properties in the canonical ensemble and to obtain exact (in statistical sense) answers to a statistical-mechanical problem. An important kind of MC simulation technique is the grand canonical Monte Carlo (GCMC) method, which allows to perform simulations in the grand canonical ensemble at constant chemical potential. ${ }^{[18]}$

The MC method is very suitable for a description of electrolyte and polyelectrolyte systems within the frame of continuum solvent models (i.e., when solvent molecules are not explicitly included in the simulations). However, for models with explicit solvent molecules, the MC method is not so efficient. The main reason is that in the liquid state, the molecules are closely packed and the fraction of the accepted MC steps becomes too small. For molecular all-atom models of the solvent, the MD simulation scheme is then more efficient.

The MD simulation technique implies a numerical solution of the Newtonian equations of motion for all atoms in the simulated molecular system. At each step of the MD simulation, the coordinates and velocities of the particles are recalculated according to equations of classical mechanics where forces are computed from some predefined expressions, called the force field. The time step is usually chosen at about $10^{-15} \mathrm{sec}$ and a typical MD simulation covers a time interval of $10^{-8}-10^{-10} \mathrm{sec}$. In addition to structural and thermodynamic properties, the dynamics of the molecular system can be studied, including time correlation functions, diffusion, and other transport properties.

At present, MD simulation is the most commonly used simulation method for studying molecules and molecular interactions in the liquid state. However, because MD implies simulation of all atoms in the system including solvent molecules, it becomes too expensive for studies of polyelectrolytes. A simplified description of polyelectrolyte systems, in terms of continuum solvent models, is still a preferable option. For such models, either the MC method or the stochastic analogue of MD-BD is used. In $\mathrm{BD}$, the solvent molecules are not explicitly accounted for, but enter the equations of motion for solute molecules by two terms: a friction force and a random force. The third term in forces describes interactions with other solute particles in the system (e.g., other ions or polyions). Because the solvent molecules are not treated explicitly in $\mathrm{BD}$, it allows simulation of substantially larger systems 
than full-atomic MD simulation. Moreover, compared with MC simulations, the dynamic properties of the ions can be determined. For example, the effect of polyions on ion diffusion can be studied.

\section{SIMULATION OF COUNTERIONS AROUND DNA WITHIN CONTINUUM SOLVENT MODELS}

\section{Evaluation of Analytical Theories}

The first attempts of computer simulations of DNA-like polyelectrolytes were made in the beginning of the 1980s using the MC method. ${ }^{[19,20]}$ More systematic studies, with varying salt concentrations and/or ion type, have been carried out later. ${ }^{[21-27]}$ The primary objective of these earlier computer simulation studies was to evaluate the applicability of analytical theories describing ionic distributions around DNA. In most of these works, the primitive model was used [i.e., the DNA was modeled as a hard body (cylinder) with a uniform distribution of surface charge, and the ions were represented as point charges or charged hard spheres]. In some of these works, a soft short-range ion-ion or ion-DNA interaction potential, ${ }^{[23]}$ or a specific location of the charges on the polyion surface $^{[22]}$ has been used. The main outcome of the early simulations was that the PB approximation is generally valid if only monovalent ions are present in the solution. In subsequent works with more detailed and more accurate (in the sense of longer runs) simulations, it was shown that deviation of the PB theory from the simulations does not exceed $10 \%$ for the ion distribution or the electrostatic potential in the relevant range of ion concentrations.

For ions of higher valency and their mixtures, the PB approximation often fails. The typical behavior is that the PB approximation underestimates the ion density in the nearest layer next to the polyion surface, which is a result of neglecting ion-ion correlations. An example is given in Fig. 1, which shows the ion density distributions for a cylindrical model of DNA in the presence of a mixture of divalent counterions and monovalent coions. One can see an increased simulated concentration of counterions compared with the PB result within about $5 \AA$ from the polyion surface. This feature may seem not large on the figure, but given the facts of the logarithmic scale of $Y$-axis and steep slope of the curve, it results in a noticeable higher affinity of divalent ions to DNA.

An additional attraction of counterions to DNA can be seen more clearly in the "integrated charge" curve, which represents ion distribution integrated from the surface of polyion to some distance $r$. The sense of this curve is the total counterion charge (per length of the polyion) within the cylinder of radius $r$ around the polyion. An example of

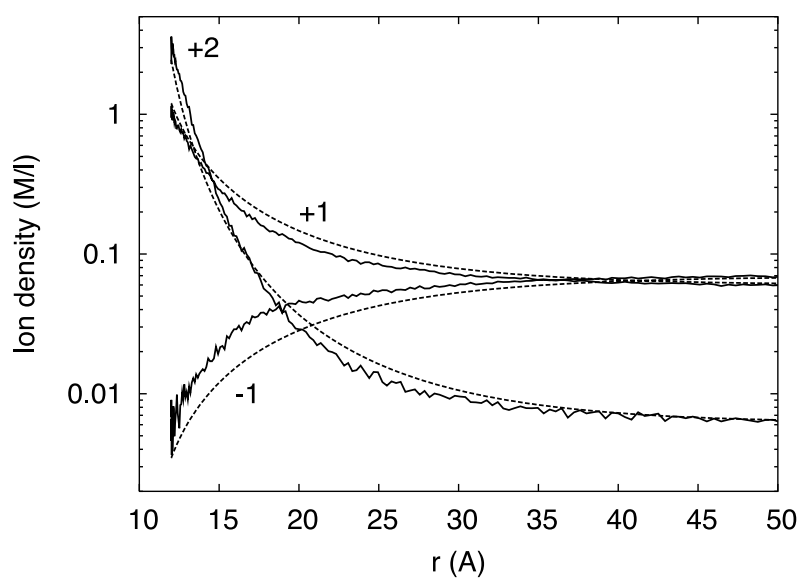

Fig. 1 Ion distribution profile around a cylindrical DNA model for a mixture of divalent and monovalent counterions and monovalent coions, obtained in MC simulation (solid lines) and PB approximation (dashed lines). Radius of all ions in MC simulations, $2 \AA$. The simulation was set up as in Ref. [37].

the integral charge curve is shown in Fig. 2 for the mixture of monovalent and divalent counterions with monovalent coins. ${ }^{[23]}$ It is seen that for the given case $\left(0.022 \mathrm{M} \mathrm{MgCl}_{2}\right.$ and $0.155 \mathrm{M} \mathrm{NaCl}$ mixture), the amount of divalent ions within $5 \AA$ from the DNA surface in the PB approximation is underestimated by about $20 \%$. In other cases (e.g., higher concentrations) deviations may reach $40 \%$ or even more.

Besides quantitative differences, computer simulations can, in some cases, predict a qualitatively different behavior as compared with the PB model. An example is the so-called "charge reversal" or "overneutralization," which may happen if the total charge of the counterions in the close vicinity of a polyion exceeds the charge of the polyion itself, which then leads to an alternating sign of the electrostatic potential. Such behavior was typically observed for divalent or higher-valence counterions under certain thermodynamic conditions, ${ }^{[25]}$ but it may happen even for monovalent ions if the salt concentration is high enough. ${ }^{[13]}$ The charge reversal cannot be obtained in the PB theory. There is no clear experimental evidence of the charge reversal, except perhaps for a rather old work by Strauss et al., ${ }^{[28]}$ who observed a cationic polyion (poly-4vinilpyridine) moving against the electric field at high salt concentration.

More accurate statistical-mechanical theories, such as the HNC or the modified PB theory, were found to reproduce properties of the ion distributions relatively well even for multivalent ions. ${ }^{[8,11,23]}$ Fig. 2, reproduced from the work of Murthy et al., ${ }^{[23]}$ shows the integral charge computed for a mixture of divalent and monovalent ions by the PB and $\mathrm{HNC}$ theories as well as by MC 


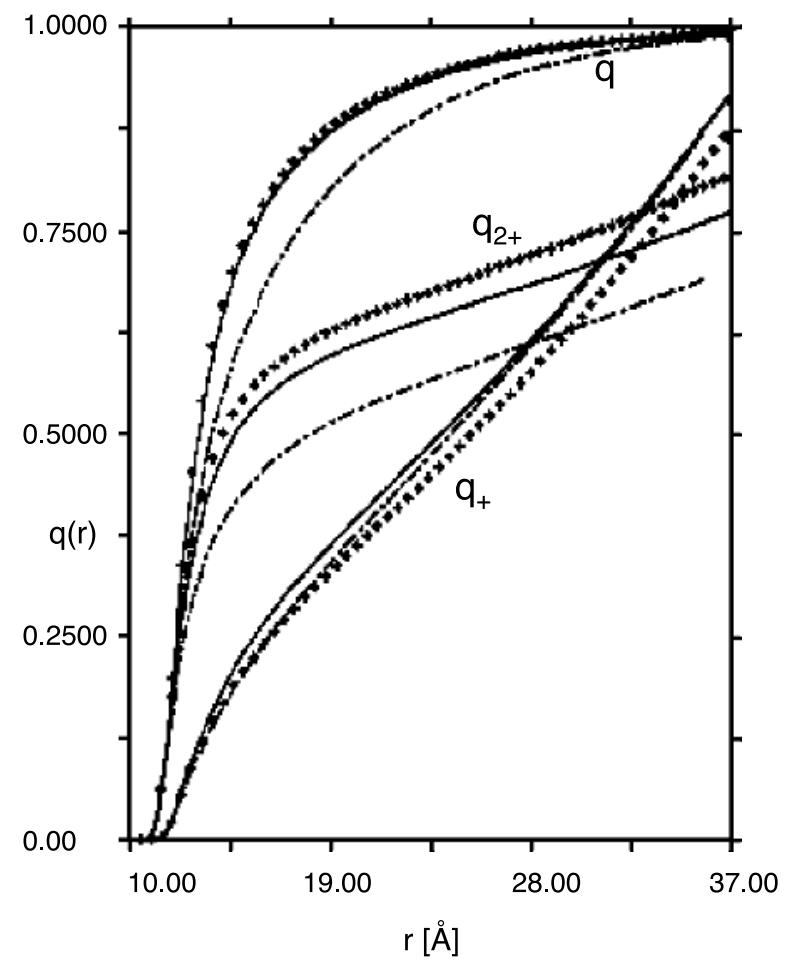

Fig. 2 Radially integrated counterionic $\left(q_{2+}\right.$ and $\left.q_{+}\right)$and total (q) charge for a $0.155 \mathrm{M} \mathrm{NaCl}$ and $0.022 \mathrm{M} \mathrm{MgCl}_{2}$ salt mixture around a cylindrical model of DNA. Points are $\mathrm{MC}$ simulation result; dash-dot lines and solid lines are PB and HNC theories, respectively. (From Ref. [23]. (C) American Chemical Society, 1985.)

simulations. Clearly, the HNC result is much closer to the simulation data than that obtained by the PB theory. The HNC theory, as well as some other more elaborated theories, are able to reproduce even such effects as charge reversal. ${ }^{[8]}$ However, these theories are difficult to apply in the case of more complicated (noncylindrical) geometries (e.g., for a model with specific location of the charges on the polyion).

Manning condensation theory is often considered as a further simplification of the PB theory. Still, it catches some basic features of ion distributions. Lamm et al. ${ }^{[27]}$ compared the fraction of electrostatically bound ions using the $\mathrm{MC}, \mathrm{PB}$, and $\mathrm{CC}$ methods. If the bound fraction of counterions is determined as those residing within a region around the DNA where the electrostatic potential is less than $k T$, the MC results are found to quantitatively reproduce the bound fraction predicted by the Manning condensation theory. Another interesting feature of ion distribution, also related to the $\mathrm{CC}$ theory, is that the concentration of counterions just next to the DNA surface depends very weakly on the salt concentration, and remains high (a few molars per liter) even at very low salt concentrations. According to the CC theory, a fraction of DNA counterions remains condensed to DNA even at infinite dilution. This feature was also confirmed by the computer simulations.

\section{Dielectric Effects}

MC computer simulations within the primitive electrolyte model imply uniform continuum dielectrics with dielectric permittivity of liquid water $(\epsilon \approx 80)$. In fact, dielectric permittivity of DNA itself is substantially lower. It is also often argued that because the water molecules around the DNA are strongly oriented in the electrostatic field of DNA, their reorientation mobility is reduced, which must make the dielectric permittivity lower than in the bulk solution. Some estimations show that the effective dielectric constant may be as low as 6-30 in the first one to two molecular layers near a DNA surface ${ }^{[29,30]}$ compared with the bulk value of about 80 . Nonuniform dielectric permittivity is a source of polarization forces, and their effect on ionic distribution is a priori unclear.

MC simulations, incorporating the effect of dielectric discontinuity ${ }^{[31,32]}$ at the polyion surface and using alternative dielectric saturation models, ${ }^{[33]}$ have investigated the effects of the assumption of a constant dielectric permittivity, finding minor effects on the counterion distributions. Moreover, the effect may be different-it may drive ions out of the DNA grooves, ${ }^{[31]}$ or, at the opposite, increase their concentration near the DNA surface, ${ }^{[32]}$ depending on the details of the DNA model (shape) and the way how the dielectric effects are incorporated.

In fact, at distances on the order of a few angstroms, the concept of dielectric permittivity is not well defined. Within the primitive model, the interaction potential is the Coulombic potential scaled by the value of the dielectric constant. This potential is an approximation of the exact solvent-mediated potentials between the ions in the solution, which in fact represents the free energy of ion-solvent interactions. From this point of view, $\epsilon$ in the interaction potential can be regarded as a parameter of the potential, which may differ from the local dielectric constant related, for example, to the water dipole fluctuation or the dielectric response. Evaluation of solventmediated potentials may be performed from the all-atom MD simulations, which showed that the effective dielectric constant - as a parameter of the effective potentialremains high even at high-enough salt concentrations and near the DNA surface. ${ }^{[33,34]}$ The effects caused by an explicit account of the solvent in computer simulations on ion distribution around the DNA will be discussed in more detail below. 


\section{Effect of Specific Distribution of Charges}

The effects caused by a specific distribution of charges on the polyion surface were studied in a number of works ${ }^{[13,31,35-38]}$ with applications to DNA. Quite evidently, a different structure of the charge distribution on the polyion may cause a rather different distribution of the ion density in the close vicinity of the polyion. Typically, one puts charges of -1 on the sites corresponding to the phosphate groups of DNA. Additionally, helical grooves may be set, mimicking the minor and major grooves of DNA. In some cases, the DNA is presented with all-atom resolution. ${ }^{[39]}$

A detailed study of different ways to mimic helical grooves on DNA was presented by Montoro and Abascal. ${ }^{[13]}$ These authors observed that specific interactions of ions with DNA (soft repulsion potential), the incorporation of the discrete charge distribution, and the grooved nature of the DNA surface may change the ion density profile around DNA considerably (Fig. 3). For example, in a "groove model" of DNA, in which explicit grooves on DNA were introduced, a double hump on the counterion density profile was observed, which even transformed to a

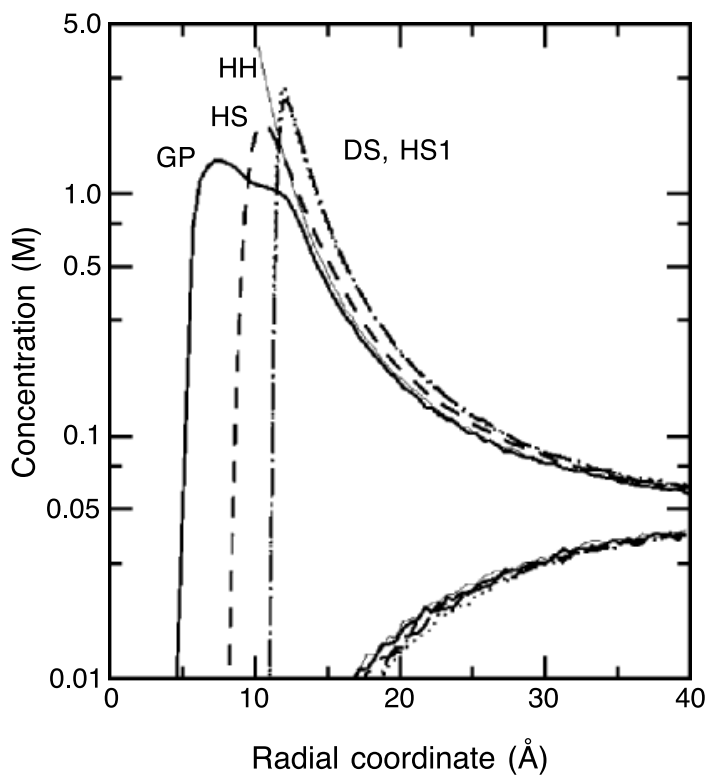

Fig. 3 Ion distribution profiles at $0.05 \mathrm{M}$ monovalent salt, calculated in MC simulations for different DNA models. HH is "'homogeneous hard cylinder," HS is "homogeneous soft cylinder' (ions interact with the polyion with repulsive $1 / r^{9}$ potential), HS1 is homogeneous soft cylinder with a displaced axis of short-range repulsion, DS is discretely charged, soft repulsion model with charges located on sites of phosphate groups, GP is grooved DNA model with explicit grooves. (From Ref. [13]. (C) American Institute of Physics, 1995.)

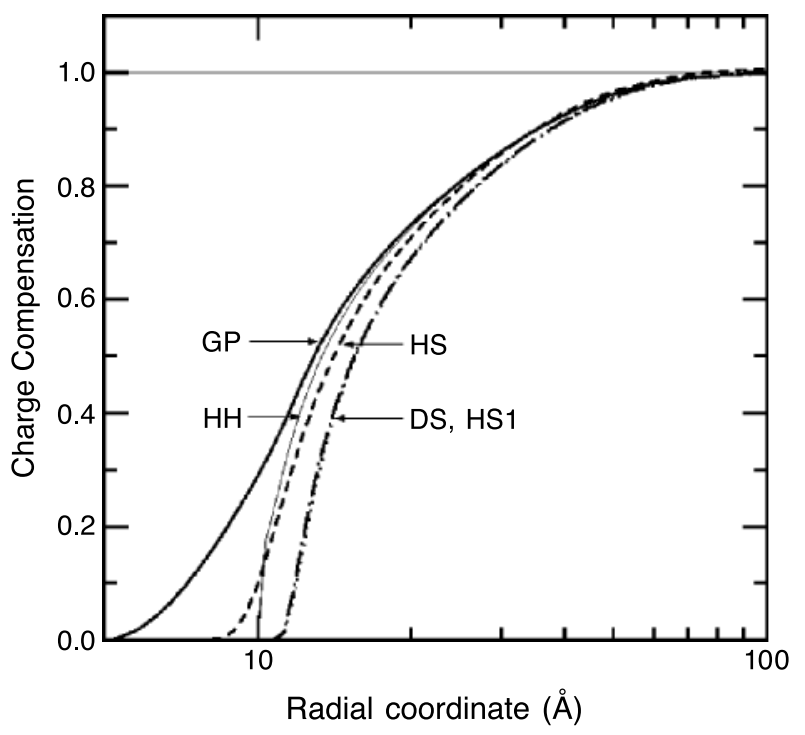

Fig. 4 Integrated charge (charge compensation) function, calculated in MC simulations for different DNA models. Notations are the same as in Fig. 3. (From Ref. [13]. (C) American Institute of Physics, 1995.)

double maximum at higher concentrations. This is in agreement with BD simulations of a full-atomic description of DNA. ${ }^{[40,41]}$ In addition, substantial penetration of counterions in the minor and major grooves was observed ${ }^{[36]}$ Enhanced concentration of counterions in the grooves (with stronger effect in the minor groove) has been observed also in several works. ${ }^{[37,38]}$ However, distribution of counterions far from the DNA depends only weakly on the structure of the DNA surface.

An MC study of a DNA model with an all-atom resolution in a continuum solvent was carried out by Mills et al. ${ }^{[39]}$ for different forms of DNA. It was found that the grooved structure of both A-DNA and the B-DNA affects the details of the ion distribution in the center region of the polyion dramatically. However, the total number of bound ions at a particular added salt value differs only slightly among the conformations.

A general conclusion from the studies on the effect of the specific charge location on DNA is that although it may affect strongly the details in ion distribution near the polyion, the distribution of counterions far from the polyion (at distances more than $20 \AA$ from the DNA axis) remains largely invariant. Furthermore, the integral properties of the ion distribution, such as the amount of ions within a certain distance around the polyion, depend very little on how the charges are located on the polyion surface. This is illustrated in Fig. 4, where the integrated charge is displayed for the same ion distributions as shown in Fig. 3. The integrated charge curves are determined 
mostly by the total charge density of the polyion and by the ion composition. The primary reason for the weak sensitivity of the global properties of the ion distribution to the details of charge location is the long-range character of electrostatic interactions, which effectively averages contributions from all the charges of the polyion and makes the total electrostatic field similar to that of a uniformly charged cylinder.

\section{Ion Competition}

The interaction of mobile ions with polyions depends strongly on the ion type, charge, and size. Counterions of higher valency are more strongly attracted to polyions than monovalent ions - that is why they force low-valency ions out from the nearest vicinity of the polyelectrolyte. The size of the ions additionally plays a role and becomes especially important in the discussion of the competition of ions of the same valency. Smaller ions can come closer to the polyion surface and lower the electrostatic energy as compared with larger ions. However, within the primitive electrolyte model, the size of ions in simulations is not directly related to their real "crystallographic" sizes. Rather, it is a parameter that, in an average way, takes into account the effective hydration shell of water molecules around the ion. Reasonable values of the ion hydration radii can be obtained by fitting to available experimental data on ion osmotic and activity coefficients. ${ }^{[42]}$ Other ways of choosing effective ion radii are discussed in a recent work by Banavali and Roux. ${ }^{[43]}$

The problem of ion competition has received much attention because of its importance in biological and technological applications. (For more information, see, for example, Ref. [44].) Typically, one determines a "competition coefficient," which has a sense of an equilibrium constant of the ion exchange process. ${ }^{[45]}$ The competition coefficient may be determined experimentally from nuclear magnetic resonance (NMR) relaxation measurements, ${ }^{[45]}$ or from direct ion exchange experiments. ${ }^{[46,47]}$ The competition parameter may be also determined from computer simulations, or other theoretical approaches in a standard manner: For a given concentration of ions of different species, the ion density profiles are calculated and from these data, the amount of ions of each species within some distance from the polyion surface is obtained. Some uncertainty arises when one tries to define which ions are "bound" and which are "free." Usually, one considers ions within some cutoff distance $r_{\mathrm{B}}$ from the surface of DNA (which is on the order of a few angstroms) as "'bound.' Paulsen et al. ${ }^{[45]}$ analyzed the dependence of the competition coefficient on the cutoff radius and found that it is almost independent of $r_{\mathrm{B}}$ if $r_{\mathrm{B}}$ is chosen in reasonable limits.
In this way, the competition of ions of several divalent counterions with $\mathrm{Na}^{+}$around the DNA was studied in the work $^{[45]}$ where a comparison of the PB model, MC simulations, and NMR experiments has been made. It was found that simulation results were closer to the experimental data than the PB results. Other studies of the ion competition effects are reported in a number of more recent MC simulations. ${ }^{[38,48,49]}$ It was shown that the PB approximation underestimates binding of higher-valency ions and that this underestimation increases with ion valency. ${ }^{[38,45]}$

In the case of concentrated polyelectrolyte systems (e.g., oriented DNA fibers in equilibrium with a bulk electrolyte solution), the situation is different. The simulation cell, in this case, cannot include the "bulk" solution, so the concentration of the ions in the bulk is not directly known. The problem can be solved by performing GCMC simulations both in the polyelectrolyte gel phase and in the bulk solution (without polyions), at the same chemical potential. ${ }^{[50]}$ The GCMC approach has been applied for studying the competition of monovalent, divalent, and trivalent ions in a series of works by Korolev et al. ${ }^{[46,47,51]}$ In these works, competition coefficients of different ions, computed in GCMC simulations, were compared with the experimental ones measured by ion exchange experiments for DNA fibers, which were stabilized by the presence of ethanol. In computer simulations, the presence of ethanol was modeled as a lower (than in pure water) dielectric constant. The competition coefficients for the ions of different valencies and sizes have been computed and a good agreement with the ion exchange measurements has been observed.

\section{Interaction of DNA with Multivalent Ligands}

Studies of DNA interacting with complex multivalent molecular ions is of considerable interest because of the role of such ions (e.g., polyamines) in living systems. Additionally, polyamines are often used as condensing agents in the preparation of condensed samples of DNA. Other interesting and important applications are related to binding of charged intercalative drugs to DNA and to protein-DNA binding.

Application of standard polyelectrolyte theories to complex multivalent ions faces additional difficulties related to the spatial distribution of the ion charges and the internal degrees of freedom. In analytical theories, these additional features are usually either ignored ${ }^{[52]}$ or treated in some approximate manner. ${ }^{[53,54]}$ In computer simulations, on the contrary, inclusion of the internal structure of the ionic ligand does not pose any principal complications. In Ref. [37], the polyamine spermidine $^{3+}$ was modeled as a chain of three monovalent ions connected by 
harmonic bonds. It was shown that because of the effects of the nonlocal charge distribution and internal degrees of freedom, the binding affinity of spermidine to DNA was reduced compared with that of a simple trivalent (metal) ion. The binding was reduced even below the predictions of the PB theory, which generally underestimates the binding affinities of higher valency ions for simple ions. Existing NMR diffusion studies qualitatively confirm this result. $^{[55]}$

The binding of an octavalent rodlike ligand to DNA in the presence of monovalent salt has been studied by Olmstedt et al. ${ }^{[56]}$ using GCMC simulations. Such highly charged ligand binds strongly to DNA, forming a stable complex. A strong reduction in the surface counterion (e.g., $\mathrm{Na}^{+}$) concentration over a region including-but extending well beyond - the location of the ligand binding site has been observed.

\section{Brownian Dynamics of Counterions Around DNA}

BD simulations allow, alongside ion distribution, computation of dynamic and transport properties of ions. The presence of a polyion makes the diffusion of ions slower than in the bulk solution, because of both the steric obstacles imposed by the polyion and the electrostatic interactions. Thus the retardation of the diffusion provides information on the binding of ions to the polyelectrolyte. An advantage of diffusion studies is that the self-diffusion coefficient is a quantity that is directly available from experiments measuring macroscopic self-diffusion.

The diffusion properties of counterions in the presence of DNA were studied by BD simulations in a number of works. ${ }^{[40,57,58]}$ The self-diffusion coefficient can also be obtained within the PB mean field approximation, using the PB-Smoluchowski diffusion model. ${ }^{[59]}$ A comparison of lithium counterion diffusion coefficients determined in BD simulations, in the PB-Smoluchowski model, and in the experiment was performed in Ref. [57]. The comparison of the two polyelectrolyte theories with the experiment clearly demonstrated the effect of ion correlations included in the BD simulations, which substantially improve agreement with the experiment.

Apart from the general interest as to how the presence of DNA affects ion dynamics, such simulations provide valuable information for the interpretation of NMR relaxation experiments. For example, BD may provide data on the time correlation function of the electric field gradients ${ }^{[40,58]}$ experienced by $\mathrm{Na}^{+}$counterions associated with DNA, for which the NMR quadrupolar relaxation of the ${ }^{23} \mathrm{Na}$ nuclei is determined by the time-dependent fluctuation of these field gradients.

\section{SIMULATIONS OF COUNTERIONS AROUND DNA WITH EXPLICIT ACCOUNT OF SOLVENT}

\section{Molecular Dynamics of DNA}

The studies of the ionic environment of DNA discussed above have been carried out within continuum solvent models. The effects of solvent and hydration can be taken into account rather straightforwardly in computer simulations by introducing explicit solvent (water) molecules. However, an all-atom description makes the simulations very time-consuming and reduces the size of the system that can be considered. At the present level of computer power, the maximum number of atoms that can be simulated long enough to obtain meaningful results is on the order $10^{4}$, which corresponds to a simulation box size of $50-60 \AA$. This size is not enough to study polyelectrolyte aspects of ion distribution. Still, all-atom simulations are extremely important for understanding ion-DNA interactions in close proximity to the DNA.

The preferable method in all-atom simulations is MD, although the first attempt to simulate such model was performed by the MC method. ${ }^{[60]}$ Earlier MD simulations of DNA ${ }^{[61-63]}$ were too short to produce information on ionic distribution. Given the diffusion of counterions around DNA on the order $10^{-6} \mathrm{~cm} / \mathrm{sec}^{2}$, at least nanosecond time scale is needed for the counterions to sample the space around DNA. Such simulations became possible from the second part of 1990s, when a large number of works on MD simulations of DNA, with full-atomic description of solvent and ions, appeared. ${ }^{[64-71]}$ In the majority of these studies, the main interest was in the DNA molecule itself, investigating DNA backbone structure and dynamics, base stacking, phosphate orientation, overall nucleic acid structure, and so on. (For more information on these issues, the reader is referred to recent reviews. ${ }^{[3,72]}$ ) Below, we shall concentrate on works dealing with MD simulations of ion distributions around DNA. Note first that results on MD simulations are dependent on the force field used. The force fields used in macromolecular simulations are usually empirically parameterized to reproduce some set of experimental results. At present, the most often used force fields for DNA simulations are $\mathrm{AMBER}^{[73]}$ and CHARMM. ${ }^{[74]}$ It was found that these force fields may give somewhat different DNA structures. ${ }^{[70]}$ However, the hydration structure and ion distribution are very similar in AMBER and CHARMM simulations. ${ }^{[71,75]}$ This is because of the fact that these force fields differ mainly by parameters describing intramolecular DNA interactions, whereas parameters describing forces between water, ions, and DNA are almost the same. 


\section{Specific Binding of lons}

A major issue in molecular dynamic studies of the ionic environment of DNA is the problem of specific ion binding to different sites on the DNA surface. Whether this binding is sequence-specific or purely electrostatic is still to be determined. X-ray experiments carried out in the 1980s have suggested that there exists a "spin of hydration" of DNA, ${ }^{[76]}$ which is a sequence of water molecules in the minor and major grooves that is impenetrable to cations. Cations, according to this picture, form a diffuse cloud around, which is defined by electrostatic interactions. However, MD simulations, made in the 1990s, have shown that $\mathrm{Na}^{+}$counterions may intrude the spin of hydration of DNA and substitute for water molecules. ${ }^{[66-68,77]}$ This was also confirmed by newer, highresolution X-ray data. ${ }^{[78]}$

The question of sequence-specific counterion binding to DNA has a principal importance to our understanding of mechanisms of DNA recognition. When counterions bind to DNA in a sequence-specific manner, they form a mosaic of nonuniform charge distribution depending on the DNA sequence. Moreover, it was supposed that direct ion binding to DNA affects the DNA structure, ${ }^{[78]}$ although this point of view is under debate. ${ }^{[79]}$ Molecular computer simulation can provide valuable information to this discussion.

Most of the mentioned MD simulations of DNA have been carried out with $\mathrm{Na}^{+}$counterions. In some works, other counterions were also studied. In Ref. [68], a comparative study of $\mathrm{Li}^{+}, \mathrm{Na}^{+}$, and $\mathrm{Cs}^{+}$around DNA was performed. It was found that these monovalent alkali ions interact with DNA in a very different manner. $\mathrm{Li}^{+}$ions bind almost exclusively to the phosphate groups of DNA. $\mathrm{Na}^{+}$ions bind prevailing bases in the minor groove through one water molecule, although a smaller fraction of ions binds directly to the bases at some specific sites (AT step in the minor groove and guanine bases in the major groove). $\mathrm{Cs}^{+}$ions bind directly to sugar oxygen in the minor groove. It was shown also that the specific character of ion bonding is, to a large extent, determined by the hydration structure of water around DNA. A stronger binding of $\mathrm{Li}^{+}$ions to DNA was also confirmed by NMR studies of the diffusion of $\mathrm{Li}^{+}$and $\mathrm{Cs}^{+}$ions in oriented DNA fibers. ${ }^{[80]}$

DNA oligomers $\mathrm{d}(\mathrm{TpA})_{12}$ in the presence of $\mathrm{K}^{+}$ counterions were simulated in Ref. [81]. Comparing with $\mathrm{Na}^{+}$counterions, a stronger preference of $\mathrm{K}^{+}$ions to the major groove has been observed.

MD of DNA with divalent ions $\mathrm{Mg}^{2+}$ and $\mathrm{Ca}^{2+}$ was performed in some recent works. ${ }^{[63,64,82]}$ However, the slow diffusion of $\mathrm{Mg}^{2+}$ ions makes it difficult to perform a reliable estimation of distribution of these ions around the DNA.
Recently, some works appeared on the distribution of multivalent polyamine ions around DNA. ${ }^{[83-85]}$ It was found that flexible polyamine molecules (spermine or spermidine) have several binding modes, interacting with different sites on the DNA in an irregular manner. That is why polyamine molecules are not seen in X-ray diffractions of DNA. Spermine ${ }^{4+}$ ions compete with $\mathrm{Na}^{+}$ions and water molecules in binding to bases in the minor groove, and they influence the structure of the DNA hydration shell in different ways. ${ }^{[85]}$

\section{Comparison with Continuum Solvent Models}

An interesting question that MD simulation could answer is: How reliable are computations within the continuum solvent model, for example, in the prediction of overall ion density around DNA? The angularly averaged density profile of counterions $\left(\mathrm{Na}^{+}\right.$mostly) has been calculated in some recent works. ${ }^{[66-68]}$ At short distances (within $5 \AA$ from the DNA surface), these density profiles are defined mainly by the details of DNA structure and hydration forces. Naturally, the distributions are very different from the density profiles calculated for the cylindrical model of DNA within the continuum solvent model (both in analytical approaches and MC simulations). Such comparison, from Ref. [68], is shown in Fig. 5, where angularly averaged distributions of different monovalent counterions around the DNA are displayed, together with the PB theory result. Introducing details of the DNA structure within the continuum dielectric model may make the counterion distribution closer to that obtained in MD simulations. For example, a maximum in ion density is

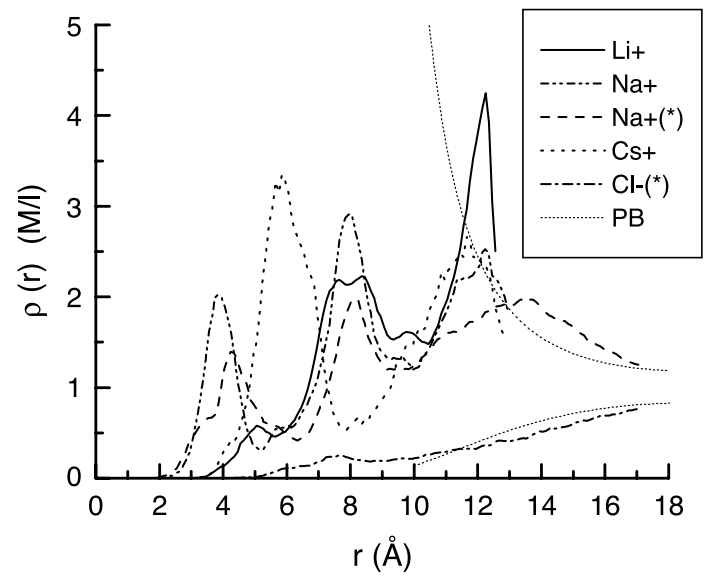

Fig. 5 Density profile of different ions around DNA obtained in all-atom MD simulations. The results marked with $\left(^{*}\right)$ are from a simulation with a larger simulation cell than the other data. The thin dotted line shows the PB results with a cell radius corresponding to the system marked $(*)$. (From Ref. [68]. Adenine Press.) 


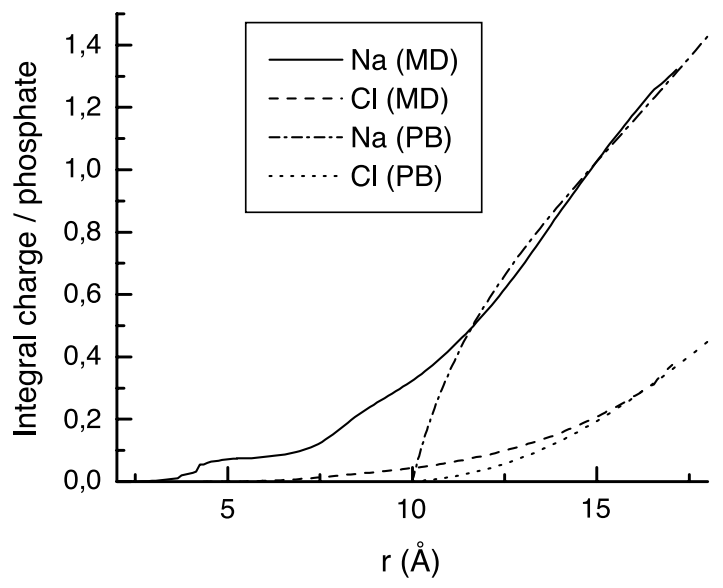

Fig. 6 Integral charge for $\mathrm{Na}^{+}$and $\mathrm{Cl}^{-}$ions per phosphate obtained in all-atom MD simulations and $\mathrm{PB}$ approximation. (From Ref. [68]. Adenine Press.)

observed at about $8 \AA$ from the DNA axis, corresponding to a high probability for counterions to be inside the DNA grooves. $^{[38]}$

On larger distances from the DNA, the ion distribution, computed in MD simulations, became more similar to that calculated in the PB theory, or obtained in continuum solvent simulations. In addition, the integral charge for $\mathrm{Na}^{+}$ions turned out to be very similar already on distances greater than $12 \AA$ from the DNA surface (Fig. 6). This means that cylindrical PB equation, despite many inherent approximations, is still able to evaluate amounts of ions that are attracted (or "nonspecifically" bound) to DNA.

\section{Multiscale Simulation Approach}

However, direct simulation of ion density in MD simulation is a tedious task and can be performed only at relatively small distances from the DNA surface. These distances do not include the true "bulk" phase, where neither water nor ions are affected by the electrostatic field of DNA. On the other hand, the problem of finding the ion distribution in the whole range (from the DNA surface to the bulk solution) is the important one. The "bulk" ion concentration in the living cell is determined by the work of ion channels in the membrane; however, only ions that are in close proximity to DNA affect their properties. Although continuum solvent models allow to compute for the ion distribution in the whole range, they contain adjustable parameters, such as the effective ion radius, and are unable to describe the effects of specific ion binding (e.g., specific binding of $\mathrm{Li}^{+}$ions to the phosphate groups).

In Refs. [33] and [34], an approach was suggested to link together the two levels of simulations of electrolyte or polyelectrolyte systems (i.e., the all-atom MD simulations and the MC simulations without explicit solvent). The main idea is that detailed all-atom MD simulations provide information on how to parameterize parameters for the continuum solvent model. In practice, as a first step, an MD simulation of size as large as can be afforded is performed. From this simulation, the radial distribution function (RDF) between ions as well as between ions and some sites of DNA is determined. Then, an inverse MC procedure is performed,${ }^{[34,86]}$ which finds the effective interaction potentials that match the RDF obtained in the MD simulations. The great benefit of effective potentials is that they can be used for simulation of the very same system but of substantially larger scale because the solvent molecules are not included. The typical behavior of the effective potentials is that they have a few oscillations at short distances, reflecting the molecular structure of the solvent, and then-at distances of more than $10 \AA-$ approach the Coulombic potential with the dielectric constant of water. ${ }^{[33,34]}$ The short-range part of the effective potentials is rather ion-specific, thus the specific features of ion-DNA interactions are automatically included in the model.

In Ref. [34], the effective potentials between different alkali ions and DNA have been determined and used for MC simulations of the ion environment of DNA. The computed ion distributions are shown in Fig. 7. One can notice clear similarities with the ion distribution obtained in all-atom MD simulations in Fig. 5. Another interesting observation is that for $\mathrm{Na}^{+}$and $\mathrm{K}^{+}$ions, the density profiles follow very closely the solution of the PB equation for distances larger than $15 \AA$ from the DNA axis (of course, they were rather different at closer distances).

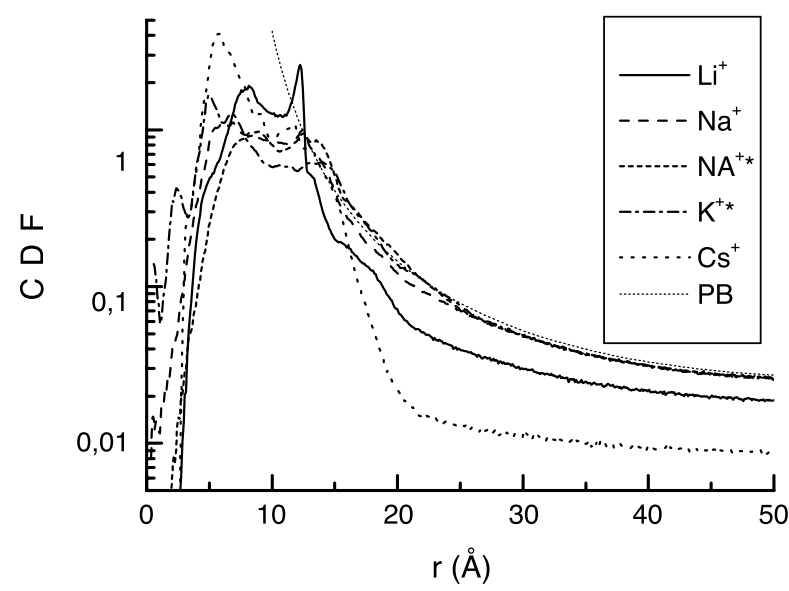

Fig. 7 Density profile of different ions around DNA obtained by MC simulation with effective solvent-mediated potentials. The notations for the curves are the same as in Fig. 5. (From Ref. [34]. American Institute of Physics. Simulation details are given in this reference.) 
For two other ions, $\mathrm{Li}^{+}$and $\mathrm{Cs}^{+}$, the deviations from the solution of the PB equation were more significant because of specific interactions of these ions with DNA. From the simulations, the relative binding affinity of these ions to DNA was determined as $\mathrm{Cs}^{+}>\mathrm{Li}^{+}>\mathrm{Na}^{+} \geq \mathrm{K}^{+}$, in agreement with the experimental results.

The multiscale modeling approach may be used to take into account solvent and hydration effects in studies of ion binding and competition, as well as of the interaction of DNA with other ligands and proteins. Work in this direction is now in progress.

\section{CONCLUSION}

The aim of the present review has been to show that computer simulation techniques are becoming increasingly important in the description of the ionic environment of DNA as well as other properties of biomacromolecular systems. Computer simulations allow us to follow the motion of every atom in the studied system, thus providing very detailed information and contributing to our general understanding of the physical mechanisms that govern the behavior of these biologically, as well as technologically, important systems. With the rapid development of computer technology, allowing larger systems to be studied on a longer time scale and enabling also a more accurate and detailed description of molecular interactions, the importance of molecular simulations in this area will grow further. Furthermore, new developments in experimental techniques including "singlemolecule" experiments, as well as an interest in more and more complicated systems related to new areas of biotechnology, biomaterials, and nanotechnology additionally enhance the importance of computer simulation methods.

\section{ACKNOWLEDGMENTS}

The author is thankful to Jose Abascal for providing electronic copies of the figures. Many thanks to Lars Nordenskiöld, Aatto Laaksonen, and Nikolai Korolev for stimulating discussions. This work was supported by the Swedish Research Council.

\section{REFERENCES}

1. Anderson, C.F.; Record, M.T. Salt-nucleic acid interactions. Annu. Rev. Phys. Chem. 1995, 46, $657-700$.

2. Jayram, B.; Beveridge, D.L. Modeling DNA in aqueous solutions: Theoretical and computer simulation studies on the ion atmosphere of DNA. Annu. Rev. Biophys. Biomol. Struct. 1996, 25, 367-394.

3. Cheatham, T.E.; Kollman, P.A. Molecular dynamics simulations of nucleic acids. Annu. Rev. Phys. Chem. 2000, 51, 435-471.

4. Lyubartsev, A.P.; Nordenskiöld, L. Computer Simulations of Polyelectrolytes; Tripathy, S.K., Kumar, J., Nalwa, H.S., Eds.; Handbook of Polyelectrolytes and Their Applications, American Scientific Publishers, 2002; Vol. 3, 309-326.

5. Fixman, M. The Poisson-Boltzmann equation and its application to polyelectrolytes. J. Chem. Phys. 1979, 70 (11), 4995-5005.

6. Manning, G.S. Limiting laws and counterion condensation in polyelectrolyte solutions. J. Chem. Phys. 1969, 51, 924-933.

7. Vlachy, V.; McQuarrie, D. A theory of cylindrical polyelectrolyte solution. J. Chem. Phys. 1985, 83 (4), 1927-1932.

8. Tovar, E.G.; Losada-Gassou, H.; Henderson, D. Hypernetted chain approximation for cylindrical polyelectrolytes. J. Chem. Phys. 1985, 83 (1), 361-372.

9. Croxton, T.; McQuarrie, D.A. The electrical double layer in the Born-Green-Yvon approximation. Mol. Phys. 1981, 42, 141-151.

10. Ramanathan, G.V. Statistical mechanics of electrolytes and polyelectrolytes: II. Counterion condensation on a line charge. J. Chem. Phys. 1982, 77 (8), 4133-4140.

11. Outhwaite, C.W. A modified Poisson-Boltzmann equation for the ionic atmosphere around a cylindrical wall. J. Chem. Soc., Faraday Trans. II 1986, 82, 789-794.

12. Vlachy, V. Ionic effects beyond Poisson-Boltzmann theory. Annu. Rev. Phys. Chem. 1999, 50, 145-165.

13. Montoro, J.C.G.; Abascal, J.L.F. Ionic distribution around simple DNA models: I. Cylindrically averaged properties. J. Chem. Phys. 1995, 103 (18), 8273-8284.

14. Klein, B.J.; Pack, G.R. Calculation of the spatial distribution of the charge density in DNA environment. Biopolymers 1983, 22, 2331-2352.

15. Cortis, C.M.; Friesner, R.A. Numerical solution of the Poisson-Boltzmann equation using tetrahedral finite-element meshes. J. Comput. Chem. 1997, 18 (13), 1591-1608.

16. Gavryushov, S.; Zielenkiewicz, P. Electrostatics of a DNA-like polyelectrolyte: Effects of solvent dielectric saturation and polarization of ion hydration shells. J. Phys. Chem., B 1999, 103 (28), 58605868.

17. Metropolis, N.; Rosenbluth, A.W.; Rosenbluth, 
M.N.; Teller, A.H.; Teller, E. Equation of state calculations by fast computing machines. J. Chem. Phys. 1953, 21, 1087-1092.

18. Wood, W.W. Physics of Simple Liquids; NorthHolland: Amsterdam, 1968.

19. Vlachy, V.; Dolar, D. Monte Carlo studies of polyelectrolyte solutions at low degrees of polymerization. J. Chem. Phys. 1982, 76 (4), 2010-2014.

20. Bratko, D.; Vlachy, V. Distribution of counterions in the double layer around a cylindrical polyion. Chem. Phys. Lett. 1982, 90 (6), 434-438.

21. Mills, P.; Anderson, C.; Record, M.T. Monte Carlo studies of counterions-DNA interactions. Comparison of the radial distribution of counterions with predictions of other polyelectrolyte theories. J. Phys. Chem. 1985, 89, 3984-3994.

22. LeBret, M.; Zimm, B. Monte Carlo determination of the distribution of ions around a cylindrical polyelectrolyte. Biopolymers 1984, 23, 271-286.

23. Murthy, C.S.; Bacquet, R.J.; Rossky, P.J. Ionic distribution near polyelectrolytes. A comparison of theoretical approaches. J. Phys. Chem. 1985, 89, $701-710$.

24. Guldbrand, L.; Nilsson, L.G.; Nordenskiöld, L. A Monte Carlo simulation study of electrostatic forces between hexagonally packed DNA double helices. J. Chem. Phys. 1986, 85 (11), 6686-6698.

25. Vlachy, V.; Haymet, A.D.J. A grand canonical Monte Carlo simulation study of polyelectrolyte solutions. J. Chem. Phys. 1986, 84 (10), 58745880 .

26. Mills, P.; Anderson, C.; Record, M.T. Grand canonical Monte Carlo calculations of thermodynamic coefficients for a primitive model of DNAsalt solutions. J. Phys. Chem. 1986, 90, 6541-6548.

27. Lamm, G.; Wong, L.; Pack, G.R. Monte Carlo and Poisson-Boltzmann calculations of the fraction of counterions bound to DNA. Biopolymers 1994, 34 (2), 227-237.

28. Strauss, U.P.; Gershfeld, N.L.; Spiera, H. Charge reversal of cationic poly-4-vinilpyridine derivatives in $\mathrm{KBr}$ solution. J. Am. Chem. Soc. 1954, 76, 59095911.

29. Hansen, W.N. The immersed double layer. J. Electroanal. Chem. 1983, 150, 133-140.

30. Young, M.A.; Jayaram, B.; Beverige, D.L. Local dielectric environment of B-DNA in solution: Result from a 14 ns molecular dynamics trajectory. J. Phys. Chem., B 1998, 102, 7666-7669.

31. Conrad, J.; Troll, M.; Zimm, B. Ions around DNA: Monte Carlo estimates of distribution with improved electrostatic potentials. Biopolymers 1988, 27, 1711-1732.

32. Jayaram, B.; Swaminathan, S.; Beveridge, D.L.;
Sharp, K.; Honig, B. Monte Carlo simulation studies on the structure of the counterion atmosphere of BDNA. Variations on the primitive dielectric model. Macromolecules 1990, 23, 3156-3165.

33. Lyubartsev, A.P.; Laaksonen, A. Osmotic and activity coefficients from effective potentials for hydrated ions. Phys. Rev., E 1997, 55 (5), 56895696.

34. Lyubartsev, A.P.; Laaksonen, A. Effective potentials for ion-DNA interactions. J. Chem. Phys. 1999, 111 (24), 11207-11215.

35. Montoro, J.C.G.; Abascal, J.L.F. Discrete charge effects in the structure of ions around polyelectrolyte model. Mol. Phys. 1996, 89 (4), 1071-1086.

36. Montoro, J.C.G.; Abascal, J.L.F. Ionic distribution around simple B-DNA models: II. Deviations from cylindrical symmetry. J. Chem. Phys. 1998, 109 (14), 6200-6210.

37. Lyubartsev, A.P.; Nordenskiöld, L. Monte Carlo simulation study of DNA polyelectrolyte properties in the presence of multivalent polyamine ions. J. Phys. Chem., B 1997, 101, 4335-4342.

38. Abascal, J.L.F.; Montoro, J.C.G. Ionic distribution around simple B-DNA models: III. The effect of ionic charge. J. Chem. Phys. 2001, 114 (9), 42774284.

39. Mills, P.A.; Rashid, A.; James, T.L. Monte Carlo calculations of ion distributions surrounding the oligonucleotide $d{\text { (atatatat })_{2}}_{2}$ in the $\mathrm{B}, \mathrm{A}$ and wrinkled D conformations. Biopolymers 1992, 32, $1491-1501$.

40. Guldbrand, L. The distribution and dynamics of small ions in simulations of ordered polyelectrolyte solutions. Mol. Phys. 1989, 67 (1), 217-237.

41. Guldbrand, L.E.; Forester, T.R.; Lynden-Bell, R.M. Distribution and dynamics of mobile ions in systems of ordered B-DNA. Mol. Phys. 1989, 67 (1), 473.

42. Triolo, R.; Grigera, J.R.; Blum, L. Simple electrolytes in the mean spherical approximation. J. Phys. Chem. 1976, 80 (17), 1858-1861.

43. Banavali, N.K.; Roux, B. Atomic radii for continuum electrostatics calculations on nucleic acids. J. Phys. Chem., B 2002, 106, 11026-11035.

44. Record, M.T.; Zhang, W.; Anderson, C.F. Analysis of effects of salts and uncharged solutes on protein and nucleic acids equilibria and processes: A practical guide to recognizing and interpreting polyelectrolyte effects, hofmeister effects, and osmotic effects of salts. Adv. Protein Chem. 1998, 51, 281353.

45. Paulsen, M.D.; Anderson, C.F.; Record, M.T. Counterion exchange reactions on DNA: Monte Carlo and Poisson-Boltzmann analysis. Biopolymers 1988, 27, 1249-1265. 
46. Korolev, N.; Lyubartsev, A.P.; Rupprecht, A.; Nordenskiöld, L. Experimental and Monte Carlo simulation studies on the competitive binding of $\mathrm{Li}^{+}, \mathrm{Na}^{+}$and $\mathrm{K}^{+}$ions to DNA in oriented DNA fibers. J. Phys. Chem., B 1999, 103, 9008-9019.

47. Korolev, N.; Lyubartsev, A.P.; Rupprecht, A.; Nordenskiöld, L. Competitive binding of $\mathrm{Mg}^{2+}$, $\mathrm{Ca}^{2+}, \mathrm{Na}^{+}$and $\mathrm{K}^{+}$ions to DNA in oriented fibers: Experimental and Monte Carlo simulation results. Biophys. J. 1999, 77, 2736-2749.

48. Das, T.; Bratko, D.; Bhuiyan, L.B.; Outhwaite, C.W. Polyelectrolyte solutions containing mixed valency ions in the cell model: A simulation and modified Poisson-Boltzmann theory. J. Chem. Phys. 1997, 107 (21), 9197-9207.

49. Ni, H.; Anderson, C.F.; Record, M.T. Quantifying the thermodynamic consequences of cation $\left(\mathrm{M}^{2+}, \mathrm{M}^{+}\right)$ accumulation and anion $\left(\mathrm{X}^{-}\right)$exclusion in mixed salt solutions of polyanionic DNA using Monte Carlo and Poisson-Boltzmann calculations of ion-polyion preferential interaction coefficient. J. Phys. Chem., B 1999, 103, 3489-3504.

50. Lyubartsev, A.P.; Nordenskiöld, L. Monte Carlo simulation study of ion distribution and osmotic pressure in hexagonally oriented DNA. J. Phys. Chem. 1995, 99, 10373-10382.

51. Korolev, N.; Lyubartsev, A.P.; Rupprecht, A.; Nordenskiöld, L. Competitive substitution of hexamine cobalt for $\mathrm{Na}^{+}$and $\mathrm{K}^{+}$ions in oriented DNA fibers. Biopolymers 2001, 58, 268-278.

52. Wilson, R.W.; Rau, D.C.; Bloomfield, V.A. Comparison of polyelectrolyte theories of the binding of cations to DNA. Biophys. J. 1980, 30, 317-325.

53. Rouzina, I.; Bloomfield, V.A. Influence of ligand spatial organisation on competitive electrostatic binding to DNA. J. Phys. Chem. 1996, 100, 43054313.

54. Stigter, D.; Dill, K.A. Binding of ionic ligands to polyelectrolytes. Biophys. J. 1996, 71, 2064-2074.

55. Andreasson, B.; Nordenskiöld, L.; Schultz, J. Interactions of spermidine and methylspermidine with DNA studied by nuclear magnetic resonance self-diffusion measurements. Biophys. J. 1996, 70, 2847-2856.

56. Olmstedt, M.C.; Bond, J.P.; Anderson, C.F.; Record, M.T. Grand canonical Monte Carlo molecular and thermodynamic predictions of ion effects on binding of an oligocation $\left(\mathrm{L}^{8+}\right)$ to the center of DNA oligomers. Biophys. J. 1995, 68, 634-647.

57. Guldbrand, L.; Nordenskiöld, L. Brownian dynamics simulation of counterion dynamics in cylindrical polyelectrolyte solution. J. Phys. Chem. 1987, 91, 5714-5718.

58. Reddy, M.R.; Rossky, P.J.; Murthy, C.S. Counterion spin relaxation in DNA solutions: A stochastic dynamics simulation study. J. Phys. Chem. 1987, 91, 4923-4933.

59. Nilsson, L.G.; Nordenskiöld, L.; Stilbs, P.; Braunlin, W.H. Macroscopic counterion diffusion in solutions of cylindrical polyelectrolytes. J. Phys. Chem. 1985, 89, 3385-3391.

60. Clementi, E.; Corongiu, G. B-DNA structural determination of $\mathrm{Na}^{+}$counterions at different humidities, ionic concentrations, and temperatures. J. Quant. Chem. 1982, 22, 595-630.

61. Seibel, G.L.; Singh, U.C.; Kollman, P.A. A molecular dynamics simulation of double-helical DNA including counterions and water. J. Am. Chem. Soc. 1985, 82, 6537-6540.

62. Swaminathan, S.; Ravishanker, G.; Beveridge, D.L. Molecular dynamics of B-DNA including water and counterions: A 140-ps trajectory for d(CGCGAATTCGCG) based on the GROMOS force field. J. Am. Chem. Soc. 1991, 113, 50275040.

63. York, D.M.; Darden, T.; Deerfield, D.; Pedersen, L.G. The interaction of $\mathrm{Na}(\mathrm{I}), \mathrm{Ca}(\mathrm{I})$, and $\mathrm{Mg}$ (II) metal ions with duplex DNA: A theoretical modeling study. Int. J. Quant. Chem. 1992, 19, 145-166.

64. MacKerell, A.D. Influence of magnesium ions on duplex DNA structural, dynamics and solvation properties. J. Phys. Chem., B 1997, 101, 646650.

65. Cheatham, T.E., III; Kollman, P. Molecular dynamics simulations highlight the structural differences among DNA:DNA, RNA:RNA, and DNA:RNA hybrid duplexes. J. Am. Chem. Soc. 1997, 119, 4805-4825.

66. Young, M.A.; Ravishanker, D.; Beveridge, D.L. A 5-nanosecond molecular dynamics trajectory for BDNA: Analysis of structure, motions and solvation. Biophys. J. 1997, 73, 2313-2336.

67. Young, M.A.; Jayaram, B.; Beveridge, D.L. Intrusion of counterions into the spine of hydration in the minor groove of B-DNA: Fractional occupancy of electronegative pockets. J. Am. Chem. Soc. 1997, 119, 59-69.

68. Lyubartsev, A.P.; Laaksonen, A. Molecular dynamics simulations of DNA in solution with different counterions. J. Biomol. Struct. Dyn. 1998, 16 (3), 579-591.

69. Young, M.A.; Beverige, D.L. Molecular dynamics simulations of an oligonucleotide duplex with adexnine tracts phased by a full helix turn. J. Mol. Biol. 1998, 281, 675-687.

70. Feig, M.; Pettitt, B.M. A molecular simulation picture of DNA hydration around A- and B-DNA. Biopolymers 1998, 48, 199-209. 
71. Feig, M.; Pettitt, B.M. Modelling high-resolution hydration patterns in correlation with DNA sequence and conformation. J. Mol. Biol. 1999, 286, 1075-1095.

72. Beveridge, D.L.; McConnel, K.J. Nucleic acids: Theory and computer simulation, Y2k. Curr. Opin. Struct. Biol. 2000, 10, 182-196.

73. Cornel, W.D.; Cieplak, P.; Bayly, C.I.; Gould, I.R.; Merz, K.M.; Ferguson, D.M.; Spellmeyer, D.C.; Fox, T.; Caldwell, J.W.; Kollman, P.A. A second generation force field for the simulation of proteins, nucleic acids, and organic molecules. J. Am. Chem. Soc. 1995, 117, 5179-5197.

74. MacKerell, A.D.; Bashford, D.; Bellott, M.; Dunbrack, R.L.; Evanseck, J.D.; Field, M.J.; Fischer, S.; Gao, J.; Guo, H.; Ha, S.; Joseph-McCarthy, D.; Kuchnir, L.; Kuczera, K.; Lau, F.T.K.; Mattos, C.; Michnick, S.; Ngo, T.; Nguyen, D.T.; Prodhom, B.; Reiher, W.E.; Roux, B.; Schlenkrich, M.; Smith, J.C.; Stote, R.; Straub, J.; Watanabe, M.; Wiórkiewicz-Kuczera, J.; Yin, D.; Karplus, M. All-atom empirical potential for molecular modeling and dynamics studies of proteins. J. Phys. Chem., B 1998, 102, 3586-3616.

75. Feig, M.; Pettitt, B.M. Sodium and chlorine ions as part of the DNA solvation shell. Biophys. J. 1999, 77, 1769-1781.

76. Drew, H.R.; Dickerson, R.E. Structure of a B-DNA dodecamer: III. Geometry of hydration. J. Mol. Biol. 1981, 151, 535-556.

77. Stefl, R.; Koca, J. Unrestrained molecular dynamics simulations of $\left[d(A T)_{5}\right]_{2}$ duplex in aqueous solution: Hydration and binding of sodium ions in the minor groove. J. Am. Chem. Soc. 2000, 122, 5025-5033.

78. McFail-Isom, L.; Sines, C.C.; Williams, L.D. DNA structure: Cations in charge? Curr. Opin. Struct. Biol. 1999, 9, 298-304.

79. McConnell, K.J.; Beveridge, D.L. DNA structure: What's in charge? J. Mol. Biol. 2000, 304, 803-820.

80. van Dam, L.; Lyubartsev, A.P.; Laaksonen, A.; Nordenskiöld, L. Self-diffusion and association of $\mathrm{Li}^{+}, \mathrm{Cs}^{+}$, and $\mathrm{H}_{2} \mathrm{O}$ in oriented DNA fibers. An NMR and MD simulation study. J. Phys. Chem., B 1998, 102, 10636-10642.

81. Auffinger, P.; Westhof, E. Water and ion binding around $\mathrm{r}(\mathrm{UpA})_{12}$ and $\mathrm{d}(\mathrm{TpA})_{12}$ oligomers-Comparison with RNA and DNA $(\mathrm{CpG})_{12}$ duplexes. J. Mol. Biol. 2001, 305, 1057-1072.

82. Bevan, D.R.; Lee, L.; Pedersen, L.G.; Darden, T.A. Molecular dynamics simulations of the $\mathrm{d}$ (ccaacgttgg $)_{2}$ decamer: Influence of the crystal environment. Biophys. J. 2000, 78, 668-682.

83. Bryson, K.; Greenall, R.J. Binding sites of the polyamines putrescine, cadaverine, spermidine and spermine on A- and B-DNA located by simulated annealing. J. Biomol. Struct. Dyn. 2000, 18, 393-412.

84. Korolev, N.; Lyubartsev, A.P.; Nordenskiöld, L.; Laaksonen, A. Spermine: An "invisible'" component in the crystals of B-DNA: A grand canonical Monte Carlo and molecular dynamics simulation study. J. Mol. Biol. 2001, 308 (5), 907-917.

85. Korolev, N.; Lyubartsev, A.P.; Laaksonen, A.; Nordenskiöld, L. On the competition between water, sodium ions and spermine in binding to DNA: A molecular dynamics computer simulation study. Biophys. J. 2002, 82, 2860-2875.

86. Lyubartsev, A.P.; Laaksonen, A. Calculation of effective interaction potentials from radial distribution functions: A reverse Monte Carlo approach. Phys. Rev., E 1995, 52 (4), 3730-3737. 


\section{Request Permission or Order Reprints Instantly!}

Interested in copying and sharing this article? In most cases, U.S. Copyright Law requires that you get permission from the article's rightsholder before using copyrighted content.

All information and materials found in this article, including but not limited to text, trademarks, patents, logos, graphics and images (the "Materials"), are the copyrighted works and other forms of intellectual property of Marcel Dekker, Inc., or its licensors. All rights not expressly granted are reserved.

Get permission to lawfully reproduce and distribute the Materials or order reprints quickly and painlessly. Simply click on the "Request Permission/ Order Reprints" link below and follow the instructions. Visit the U.S. Copyright Office for information on Fair Use limitations of U.S. copyright law. Please refer to The Association of American Publishers' (AAP) website for guidelines on Fair Use in the Classroom.

The Materials are for your personal use only and cannot be reformatted, reposted, resold or distributed by electronic means or otherwise without permission from Marcel Dekker, Inc. Marcel Dekker, Inc. grants you the limited right to display the Materials only on your personal computer or personal wireless device, and to copy and download single copies of such Materials provided that any copyright, trademark or other notice appearing on such Materials is also retained by, displayed, copied or downloaded as part of the Materials and is not removed or obscured, and provided you do not edit, modify, alter or enhance the Materials. Please refer to our Website User Agreement for more details.

\section{Request Permission/Order Reprints}

Reprints of this article can also be ordered at http://www.dekker.com/servlet/product/DOI/101081EENN120009094 